\title{
Design and Implementation of a Real-time Positron Imager
}

\author{
Pranab Sabitru Naik*, Christopher David Beling, Stevenson Fung \\ Department of Physics, University of Hong Kong, Pokfulam Road, Hong Kong
}

\begin{abstract}
In this paper we are going to present the first real-time S-parameter positron imager. This is a useful tool in solid state technology for mapping the lateral defect types and concentrations on a material sample. This technology has been developed for two major categories of researchers, the first being those that have a focused low energy positron beam and second those that do not. Here we describe the design and implementation of a real-time automated scanning system that rasters a sample surface with a $0.5 \mathrm{~mm}$ diameter positron source (or beam focus) so as to give an S-parameter image of a sample. The source (or beam) rasters across a region of a semiconductor sample in rectilinear motion while gamma ray energies $\mathrm{E}_{\gamma}$ are processed using a standard $\mathrm{HP}$ Ge spectroscopy system and a 14 bit nuclear ADC. Two other $\mathrm{ADCs}$ are used to obtain the $\mathrm{x}$, y coordinate data corresponding to each event by storing voltage pulses from the $\mathrm{x} \& \mathrm{y}$ stepper motor drives (or saddle coil currents) gated with the event pulses. Using these event data triplets (x, y, $\mathrm{E}_{\gamma}$ ) the Sparameter is computed in real time for each pixel region and is used to refresh a color image display on the screen coordinates. Optimal use is made of processing time and the system resources. This user-friendly system is efficient for producing high resolution S-parameter images of the sample. (patent pending 2003)
\end{abstract}

Keywords: Real-time imaging, S-parameter, white-noise, optimal time, defect profiling, positron annihilation spectroscopy, positron beam, resolution switching, user-friendly.

\section{INTRODUCTION}

Positrons are well known for their sensitivity to vacancy type defects in solids. In the Doppler broadening spectroscopy technique the shape of the $511 \mathrm{keV}$ positron annihilation line as represented through the S-parameter provides a helpful measure of neutral and negatively charged vacancy defects when referenced to the S-parameter of non-defected material. The S-parameter is a standard and simple way in positron Doppler broadening spectroscopy for monitoring the shape of the annihilation line ${ }^{[1]}$. It is calculated by first defining a central region of the annihilation line and then dividing the events in this region by the total number of annihilation events. S-parameter imaging of a wafer surface by lateral scanning a point source of positrons across the wafer surface can provide useful information on the lateral variation of bulk defect density in the wafer. Such scans have usually been made in the past by moving a radioactive source ${ }^{[2]}$ or a positron beam ${ }^{[3,4]}$ point by point across a wafer. In this method, the beam or radioactive source is kept fixed and one point on the sample is exposed to that radiation for some fixed time (typically a few minutes) and all annihilation events are then recorded for that point before moving on to the next. The next point is then exposed to the positron radiation and so on until the whole sample surface has been scanned. The whole data set is finally processed off-line and is finally plotted in the form of an S-parameter graph using a standard plotting software.

Some of the major draw backs of this standard method are: (i) The image can only be visualized after the completion of the whole surface scanning, which takes typically a day or so for storing sufficient information to produce a good quality image. Using this method it is quite possible to collect data for a longer than necessary period if the Sparameter features of the sample turn out to have good S-parameter contrast. In some cases for which a sample provides a prominent defect profile less data at each pixel point may still make possible some required feature identification. (ii) Analyzing the image with any graphing software is always static. In such a system, one can't dynamically interact with the visualization process. (iii) In this method, there is no global monitoring of the image during data taking. For example the right justification of the sample with respect to the scanning source/beam may be in error and such an error could only be picked up after a completed rectilinear scan had been made thus causing time to be wasted in repositioning the sample. (iv) In this method, even those research groups having a positron beam are restrained to move the sample using a mechanical system with the resulting possibility of some mechanical backlash.

In contrast to the above, the real-time imager presented here works not by sequential point-by-point accumulation of data, but by parallel rapid and continuous rastering of the whole sample surface with information at each pixel location being built up simultaneously. The system is supported by specially designed imaging software that

\footnotetext{
* pranabsabitru@hkusua.hku.hk ; phone +852 91239577 ; fax +852 28598972 ; hku.hk/psnaik/
} 
allows the user to monitor the image build-up online in a dynamic and interactive mode that facilitates the formation of a good quality image of the sample in the shortest possible time. This imager has many more features that will be discussed in the next sections. After successfully testing this system with a small diameter $5-20 \mu \mathrm{Ci}{ }^{22} \mathrm{Na}$ source, the system is now being applied for use in conjunction with a sub-millimeter diameter positron beam.

\section{THE IMAGER}

The S-parameter imaging system consists of three major sections, namely the ${ }^{22} \mathrm{Na}$ source scanning apparatus, the pulse processing electronics and the dedicated system software. The imager is basically developed with the use of a radio active positron source and then applied to the case where the positron source is a focused low energy positron beam. For this reason the basic imager is first described and this is then followed by a description of the positron beam application.

\subsection{Basic S-Parameter Imager}

\subsubsection{The source and scanning apparatus:}

This apparatus is shown in Fig.1. The source is comprised of a $0.5 \mathrm{~mm}$ diameter $5 \mu \mathrm{Ci}{ }^{22} \mathrm{Na}$ in the form of $\mathrm{NaCl}$ encapsulated in $8 \mu \mathrm{m}$ thick Kapton foil. The source is supported by a thin plastic sheet that is attached to an $\mathrm{Al}$ support frame. The $\mathrm{Al}$ support frame is connected to an X-Y stepper motor drive. The source is suspended $0.5 \mathrm{~mm}$ above the wafer surface. [Other types of source designs were considered - such as ones involving collimation of positrons by $\mathrm{Al}$ or heavier materials that may allow some screening of annihilations coming directly from the source material itself, but all were found to have some disadvantage. Finally it was decided that a source with no backing material and no collimation provided the best results. Positrons ejected in the direction opposite that of the wafer traveled tens of $\mathrm{cm}$ in the air and the fraction of annihilations received from such positrons was thus minimized through the inverse square law.] During the scanning motion the source to center of detector distance varies between 5 and $6 \mathrm{~cm}$ causing slight variations of count rate which have no significant effect on the detectors energy resolution.

The source is moved in rectilinear motion (see Fig.1) by a couple of stepper motors which operate linear screw drives. The stepper motor drives are controlled by an independent computer. LVDTs (Linear Variable Differential Transformers) are attached to the base of the support frame so as to provide voltages proportional to both $\mathrm{x}$ and $\mathrm{y}$ motions.

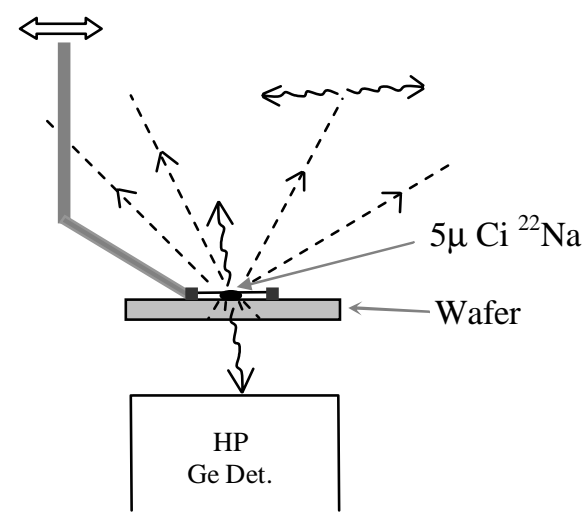

(a)

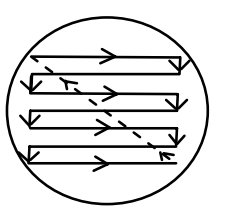

(b)

Fig. 1. Source and scanning setup together with wafer and detector (a) side view (b) top view of wafer showing motion of the source

Annihilation photons (predominantly from the wafer) were detected using a HP Ge detector of $20 \%$ efficiency. The data rate into the annihilation line was limited to 1,500 positron annihilation events per second after optimizing the detector to source distance.

\subsubsection{Electronic instrumentation}

Fig.2 depicts the block diagram of the electronic instrumentation. As the positron source rasters across the wafer surface, the $\mathrm{x}$ and y LVDTs provide DC voltages proportional to the displacement in these directions. These voltages are converted to pulses of $2 \mu \mathrm{s}$ width using two linear gates that are enabled from a TSCA output, which in turn is derived from annihilation photons that give energy signals in a narrow window around the $511 \mathrm{keV}$ energy band. The advantage of this technique is that for every energy event within the defined window there will always be an associated $\mathrm{x}$ and $y$ pulse, the amplitude of which gives the position of the source at the time of the event. The annihilation photon energies $\mathrm{E}_{\gamma}$ together with the $\mathrm{x}$ and $\mathrm{y}$ pulse signals are processed using a 14 bit nuclear ADC (i.e. peak search and hold ADC). Here the use of a TSCA (Timing Single Channel Analyzer) and the S.Amp (Spectroscopy Amplifier) are the same as used in any standard nuclear instrumentation setup. 


\subsubsection{The system software}

Using the event data triplets $\left(\mathrm{x}, \mathrm{y}, \mathrm{E}_{\gamma}\right)$ the $\mathrm{S}$-parameter is computed in real time for each pixel region and is used it to refresh a colour image display on the screen coordinates. The details of the software will be published else where. However we can briefly mention some of the features of the software as : (i) The software is efficient for real-time data acquisition and image processing. (ii) The software possesses algorithms which handle computation of all physical parameters in optimal time. (iii) The system software possesses embedded specialized algorithms and techniques providing the user with adequate freedom for analyzing various aspects of the image in order to obtain a clear inference of the defect profile. (iv) The software comprises efficient switching of the number of pixels used for the image since for short data accumulation times there is insufficient information available to support a large dimension image. (v) It also comprises diagnostic tools for monitoring the system setup and overall performance.

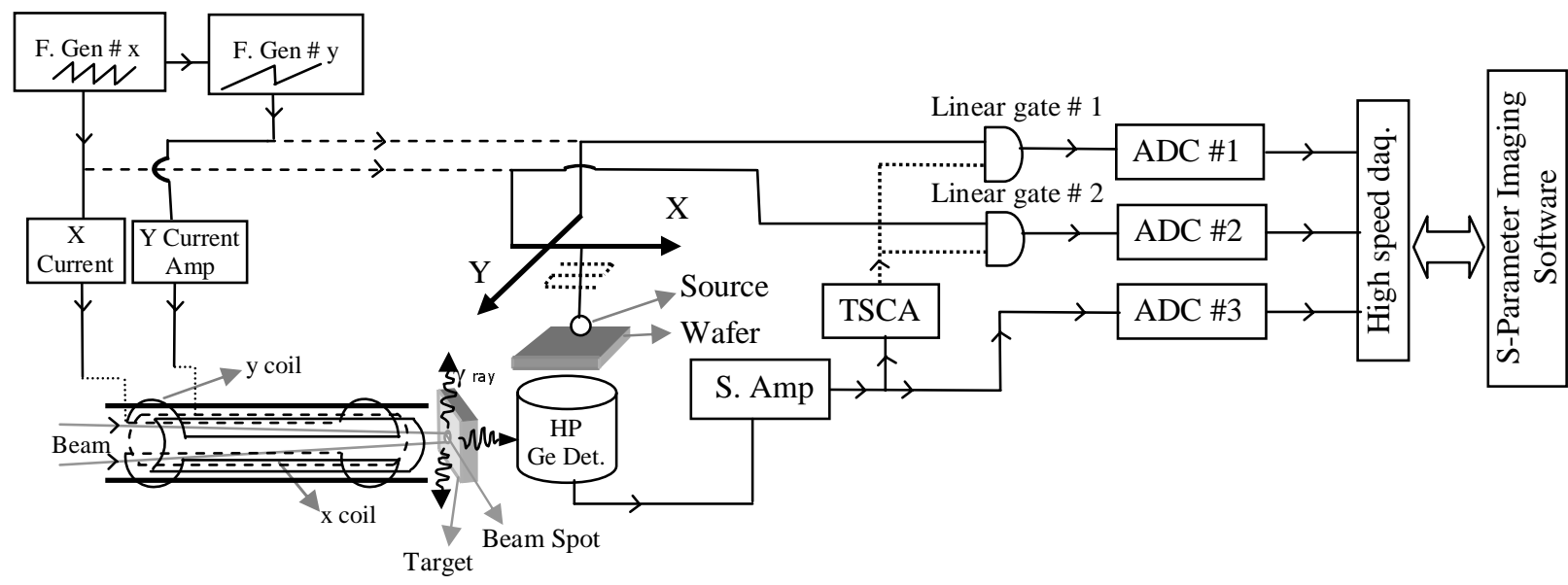

Fig. 2. The Electronics setup.

\subsection{Application to a Focused Positron Beam}

The imager system as described finds easy extension to use with a focused monoenergetic positron beam. Here the term "focused" means that the positron beam diameter should ideally be $1 \mathrm{~mm}$ or less. This focused beam spot essentially replaces the small diameter positron source. The xy scanning by the dual stepper motor system is replaced by a rastering motion of the positron beam, which is now achieved by the displacement of the charged particle beam using appropriate time-varying (saw-tooth on y and triangular on $\mathrm{x}$ ) magnetic fields supplied by $\mathrm{x}$ and $\mathrm{y}$ deflection coils. Fig. 2 also shows in block diagram this complete plan of the S-parameter imager as used with a positron beam system. Due to the fairly strong ( 100G) axial magnetic field normally used in low energy positron beams the most appropriate form of deflection is adiabatic deflection in which a small sideways magnetic field causes a small change in the direction of net magnetic field and the positron motion down the beam. For the HKU beam conventional saddle coil windings of $70 \mathrm{~cm}$ length, capable of providing a deflection field of 1 Gauss are employed. Each pair of coils has a resistance of $7 \Omega$ which are used in conjunction with current amplifiers that can supply up to $1 \mathrm{~A}$. The $\mathrm{X}$ coils are being fed uniform triangular waves (Freq $=50 \mathrm{~Hz}, \mathrm{Ampl}=5 \mathrm{~V}$ ) from a signal generator. The y coil pairs are fed with a 'saw-tooth' ramp voltage (Freq= $1 \mathrm{~Hz}, \mathrm{Amp}=5 \mathrm{~V}$ ) from another signal generator. All the parameters of the signals can be varied/controlled as per the beam deflection needs of the user. As shown in Fig.2 the $\mathrm{x}$ and y signals are being fed to the nuclear ADC along with the amplified energy output ' $\mathrm{E}_{\gamma}$ ' of the detector so as to keep track of the position coordinates of each event. Events are thus digitized as 'triplets' $\left(\mathrm{x}, \mathrm{y}, \mathrm{E}_{\gamma}\right)$ with every $\mathrm{E}_{\gamma}$ that falls within the $511 \pm 10 \mathrm{keV}$ energy band forming a triplet and those falling outside not. The 'data ready' signals from each of the three ADC channels are put through a triple AND gate so that only when a data triplet is present does the data acquisition card look at the event. This allows the software to only be employed efficiently in processing only valid annihilation line events.

\section{PRELIMINARY RESULTS AND DISCUSSIONS}

A number of surfaces have been imaged using the S-parameter scanner system and some of these are reported here. The first may be seen in Fig.3 which in (a) shows the sample being scanned by the radioactive source scanner. It consists of a $3 \mathrm{~cm} \times 5 \mathrm{~cm}$ copper block that has the letters HKU embedded into it in aluminum plate. The S-parameter image which is formed after a day of data collection is shown in Fig.3b. The letters HKU are clearly seen. 
Another example of imaging with a radioactive source is shown in Fig.4. Fig.4a is a photo of the sample being imaged. It consists of a $\mathrm{Si}$ wafer with a $0.5 \mathrm{~mm}$ thick kapton (polymide) film lightly glued to the front in the shape of an ' $\mathrm{e}^{+}$' symbol. Fig.4b shows the result of scanning for $24 \mathrm{~h}$. The ' $\mathrm{e}^{+}$' shape is clearly with good contrast.

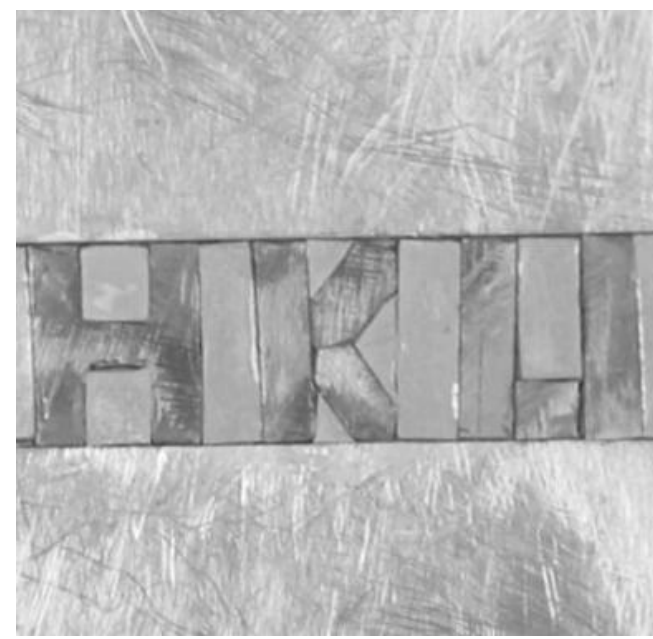

(a)

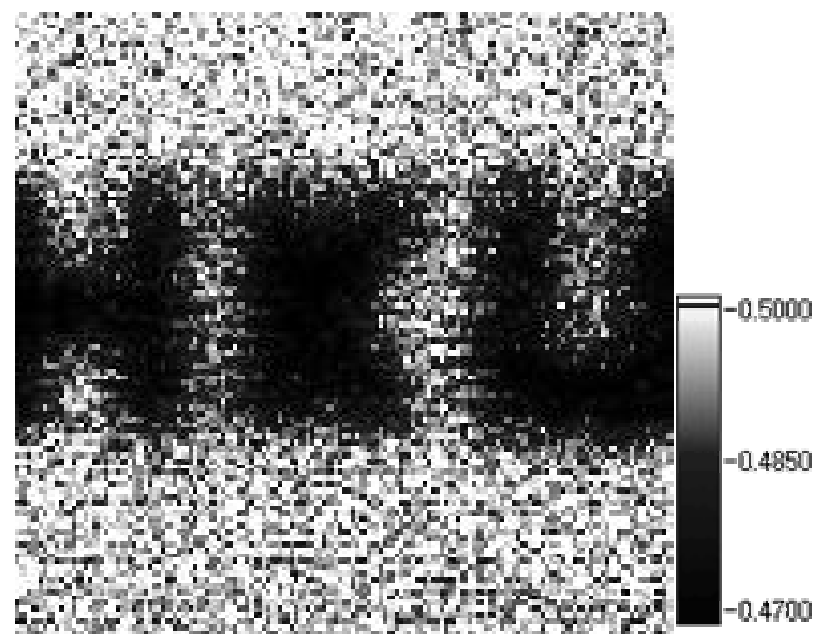

(b)

Fig. 3. Copper engraved in Aluminum plate. (a) photograph of the sample and (b) the S-parameter image.

A third example is shown in Fig.5 which shows the result of scanning an $\mathrm{n}$-type GaAs wafer. It is here that the usefulness of the positron in detecting regions of high defect concentration is seen. The granulation seen in the Sparameter image (Fig.5b) is not the typical 'white-noise' that one would expect from the statistical fluctuations of the Sparameter. There are regions such as those shown where the S-parameter is consistently high (more than 5 standard deviations) over a region of a cluster of pixels. Such regions can only be interpreted as being regions of high point defect density. Indeed the value of the S-parameter increase ( $5 \%)$ tends to suggest these regions contain divacancies or vacancy clusters.

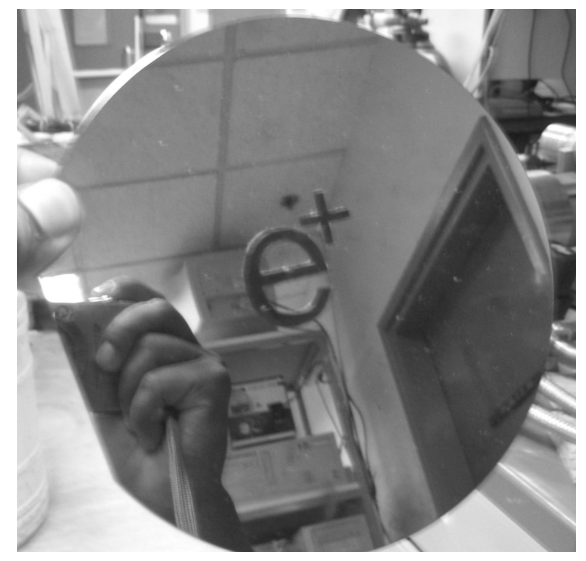

(a)

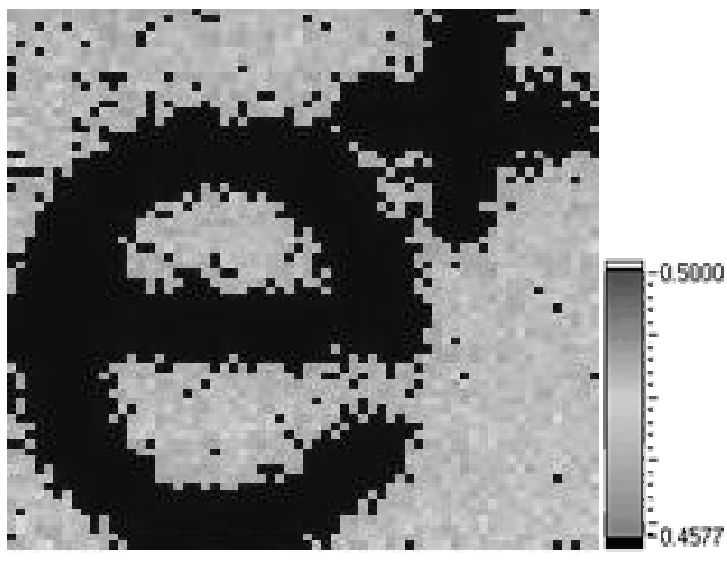

(b)

Fig. 4. Kapton on a silicon background. (a) a photograph of the sample and (b) the S-parameter image.

Work is currently in progress to successfully construct an S-parameter imaging system based on a low energy positron beam. The system comprises a novel hybrid optics that allows both efficient collection of moderated positrons and millimeter focusing at the target ${ }^{[5]}$. Positrons are collected from the tungsten moderator using the standard electrostatic Soa geometry in a magnetic field free region, after which they pass through a focusing lens that accelerates them into the main transporting magnetic field at a beam energy of $7.5 \mathrm{keV}$. The beam is transported in a series of repetitive nodes (foci) and antinodes, the last node of which is brought to focus on the target. During this transport the 
beam is filtered with an ExB velocity filter and deflected with the $\mathrm{x} y$ deflection action of the saddle coils as described above. The rastering action of the submillimeter positron focus at the target will allow samples of up to $15 \times 15 \mathrm{~mm}^{2}$ area to be imaged. The main advantage of this focused beam S-parameter imager will be that the positron beam operator will be able to know with certainty where the positrons in the beam are being focused on the sample. This will be

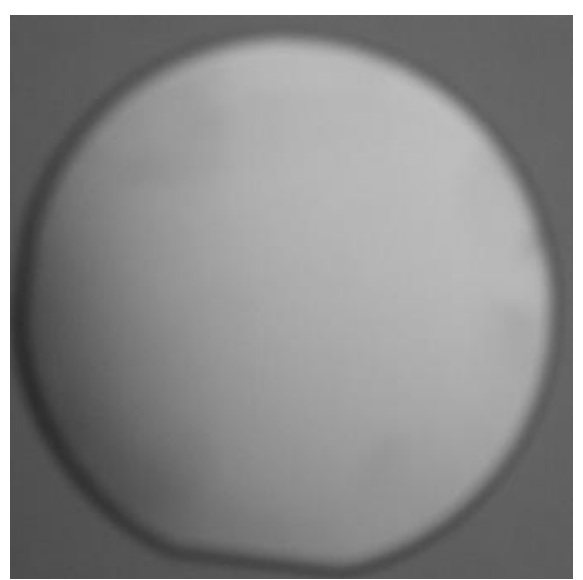

(a)

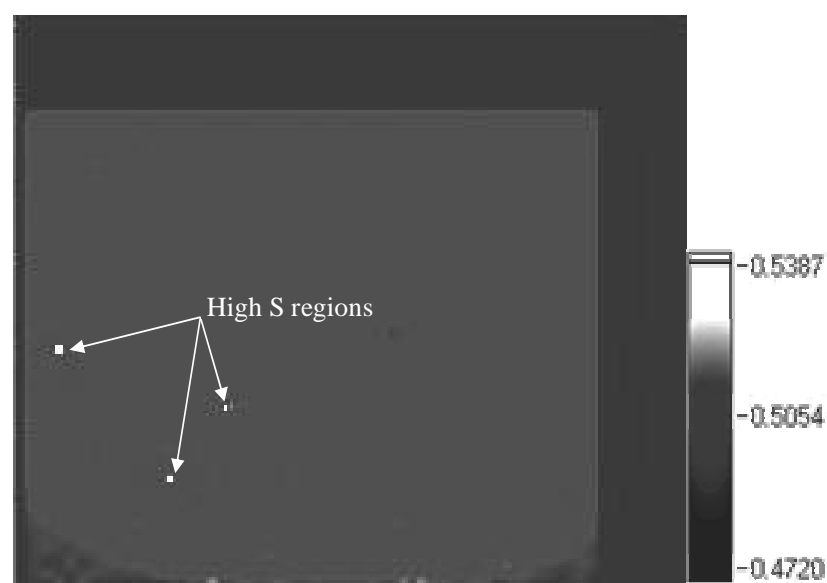

(b)

Fig. 5. n-type GaAs wafer. (a) Photograph of the wafer. (b) S-parameter image of the bottom left section of the wafer. Regions of high $\mathrm{S}$ are indicated.

particularly valuable when small $(<1 \mathrm{~mm})$ diameter Schottky diodes and photovoltaic devices are being studied since, knowing the $\mathrm{x}$ and $\mathrm{y}$ steering currents required to center the beam on the device, the beam operator will be able to optimally direct the full intensity of positrons at the active area of the device. We hope to report on the first such positron beam image in the near future.

\section{ACKNOWLEDGEMENTS}

This work was supported under grants HKU 1/00C and HKU7091/00P of the RGC of the Hong Kong Special Administrative Region. We are extremely thankful to Mr William Cheung for his hard work to setup the beam. Our sincere thanks are due to Mr Simon Hui and Mr Daviz Zhang for their co-operation and company. The help of Mr Liu of TSC, HKU is gratefully acknowledged.

\section{REFERENCES}

[1] J.L. Campbell, “Annihilation gamma-ray line shape parameters”, Appl. Phys., Vol 13, p365 (1977).

[2] G Dlubek, O Brummer, F Plaza, P Hautojärvi, J. Phys. C, "Positron study of native vacancies in doped and undoped GaAs", Semiconductor Phys., Vol 19, p 331 (1986).

[3] P.G. Coleman, "Positron Depth Profiling”, Materials Science Forum, Vols. 363-365, p 420 (2001).

[4] U. Mannig, K. Bennewitz, H. Bihr, M. Haaks, W. Sigle, C. Zamponi, K. Maier, "Defect production by the TEM beam-the first application of the positron microprobe", Applied Surface Science, Vol 149, p217 (1999).

[5] C.K.Cheung, P.Y.Kwan, Y.Y.Shan, P.S.Naik, C.D.Beling, S.Fung,"Observation of Summillimeter Positron Focusing Using a Hybrid Lens", Material Science Forum(in press). 